\title{
KN05 | 40 Years Of Ccp4: Where We Are, How We Got Here And Where We Are
}

\section{GoING}

Noble, Martin (Newcastle University, Newcastle Upon Tyne, GBR); Wilson, Keith (York University, York, GBR); Dodson, Eleanor (York University, York, GBR); Krissinel, Eugene (Science \& Technology Facilties Council, Didcot, Oxfordshire, GBR)

Collaborative Computational Project 4 (CCP4) was founded in 1979 with the aim of supporting computational macromolecular crystallography $(\mathrm{MX})$ by promoting communication among developers, sharing algorithms as they were implemented, and promoting interoperability of software and data. From modest beginnings, CCP4 has contributed hugely to the growth and maturation of $M X$, enabling this discipline to obtain a central role in the investigation of mechanistic cell biology and in the exploitation of scientific insights in biotechnological and pharmaceutical applications. This talk will review some of the history of CCP4, and describe the current status and ambitions of the project as it continues to address important challenges during its fifth decade of activity. 